\title{
O ESTADO DE BEM-ESTAR SOCIAL E AS POLÍTICAS PÚBLICAS DE SAÚDE DIRECIONADAS AO CÂNCER GINECOLÓGICO
}

\section{ARTIGO DE REVISÃO}

FERNANDES, Ingridy Tayane Gonçalves Pires ', FERREIRA, Márcia Zotti Justo 2, MAGALHÃES, Plinio Regino ${ }^{3}$, FLORES, Péricles Cristiano Batista ${ }^{4}$, CONCEIÇÃO, Keila Martins da ${ }^{5}$, CAETANO, Solange Aparecida ${ }^{6}$, LEONI, Elaine Aparecida ${ }^{7}$, VIEIRA, Valdemir ${ }^{8}$, FRINHANI, Jamille ${ }^{9}$, FRANCESCO, Daniela Simões Silva $\mathrm{Di}^{10}$

FERNANDES, Ingridy Tayane Gonçalves Pires. Et al. 0 estado de bem-estar social e as políticas públicas de saúde direcionadas ao câncer ginecológico. Revista Científica Multidisciplinar Núcleo do Conhecimento. Ano 06, Ed. 06, Vol. 15, pp. 19-

${ }^{1}$ Mestre.

${ }^{2}$ Doutora.

${ }^{3}$ Especialista.

${ }^{4}$ Mestre.

${ }^{5}$ Especialista.

${ }^{6}$ Especialista.

${ }^{7}$ Especialista.

${ }^{8}$ Mestre.

${ }^{9}$ Especialista.

${ }^{10}$ Especialista.

RC: 89583

Disponível em: https://www.nucleodoconhecimento.com.br/saude/cancer-ginecologico 
32. Junho de 2021. ISSN: 2448-0959, Link de acesso: https://www.nucleodoconhecimento.com.br/saude/cancer-ginecologico, DOI: 10.32749/nucleodoconhecimento.com.br/saude/cancer-ginecologico

\section{RESUMO}

Este estudo propõe-se uma reflexão sobre o estado de bem-estar social e relevância das políticas públicas direcionadas ao câncer ginecológico, aprimorando a assistência ao bem-estar da mulher, que engloba a atenção primária, secundária e terciária no decorrer das fases nos ciclos feminino. Para isso, buscou-se uma interpretação histórica básica sobre o Welfare State apresentando suas principais características, com o objetivo de conceituar as teorias, os períodos e suas fases ao longo dos anos, tanto no exterior como no Brasil, esboçando assim o seu desenvolvimento em questões voltadas às políticas de proteção social, com intuito de maximizar as políticas voltadas a saúde da mulher em oncologia, promovendo dessa forma a qualidade de vida na sua sobrevida. Esta discussão bibliográfica descritiva, teve uma abordagem qualitativa, fundamentando-se em estudos sobre o estado do bem-estar social em conjunto com as ações das instâncias governamentais e organizações sociais, descrevendo sua trajetória histórica desde a década de 30. Buscou-se nesta revisão também evidenciar as dimensões que contribuem para a dignidade da mulher e sua melhoria em qualidade de vida, demonstrando que essas políticas possibilitam sua reabilitação e reinserção em sua vida social.

Palavras-Chave: Estado de bem-estar social, Qualidade de vida, Câncer Ginecológico.

\section{INTRODUÇÃO}

Esse trabalho tem o objetivo de apresentar o Wefare State (WS), apresentando as abordagens e periodização tanto no exterior quanto no Brasil, assim como também apresentar os debates teóricos emergentes do estado capitalista no período pós segunda Guerra Mundial, por meio de regimes políticos de proteção social. E como $\mathrm{RC}: 89583$

Disponível em: https://www.nucleodoconhecimento.com.br/saude/cancer-ginecologico 
objetivos específicos versar as dimensões das políticas públicas de saúde frente a ações voltadas à qualidade de vida das mulheres acometidas e associar aos direitos e à dignidade da mulher; e propor ações de qualidade de vida nesse âmbito.

O desenvolvimento do estado de bem-estar social no Brasil, positivada na Constituição Federal de 1988, atribui ao Estado o encargo de proteção social, podendo ser em alguns casos residual, meritocrático e institucional, como promoção e proteção de riscos inerentes a vida com acesso universal, gratuito e igualitário.

Esse dever de proteção social, exige que a administração pública seja organizada para prestação de ações e de serviços, por meio de políticas públicas, para garantia de cidadania, pela regulamentação, fiscalização e controle, em todos os ciclos da vida, como na sobrevida de mulheres acometidas pelo câncer ginecológico, no qual se destaca o câncer de colo de útero.

De acordo com Fleck (2008), a avaliação de qualidade de vida, define o estado de saúde em si, como um conjunto de níveis de funcionalidade ou capacidades intrínsecas ao indivíduo, tendo como consequência os fatores de risco para a saúde, sendo afetados pelo mundo externo, e de que maneira o indivíduo realiza suas tarefas e ações. O ambiente também é responsável por causar alterações no estado fisiológico de um indivíduo, porém o estado de saúde em si está diretamente ligado aos fatores internos de cada pessoa.

O Instituto Nacional de Câncer (INCA) e o Ministério da Saúde (MS) denotam, o câncer do colo do útero, como o terceiro mais comum entre as mulheres e como a quarta causa de óbitos por câncer na população feminina, reconhecendo-se que, na prática clínica oncológica, a paciente experimenta variações da sintomatologia do início até o término do tratamento, além das sequelas irreversíveis.

Conforme salienta Arretche, (1995) o trabalho não remunerado da sociedade é exercido especificamente pelas mulheres, sendo assim, do ponto de vista da provisão 
social, os sistemas de proteção social refletem na estrutura de poder dominante das famílias a representação feminina.

Complementarmente, Viana (2005) diz que as questões que envolvem a proteção social são transpostas nas relações de dependência geradas pela desigualdade social desencadeando a formulação de tipos mais significativos de política e proteção social.

Este modelo assistencialista em sua ação, não é nem político, e nem social; reproduz aos assistidos em todos os ciclos da vida, a exclusão social, principalmente causada pelas sequelas de uma doença crônica, tem a sua juridicidade encontrada na bondade das pessoas.

Assim, o estado de bem-estar social, apresenta diversos conceitos e tipologias, pois em sua análise requer uma observação a partir de sua origem, apresentando diferentes características e aspectos de explicação como econômico, político, social e institucional (DRAIBE, 1993).

Importante esclarecer que Viana e Levcovitz (2005) trata esta análise do estado de bem-estar social, como uma visão histórica que está ligado a diversos fatores como institucionais, econômicos e políticos que condicionam esta expectativa a partir da região e do momento histórico.

Deste modo, a proteção social em suas inúmeras vertentes, necessita-se não apenas de sua análise teórica, mas do reconhecimento em sua aplicabilidade legitima como direito, uma vez que, dentro do contexto de influências econômicas, ambientais e sociais relaciona-se ao câncer de colo do útero, tratando-se do impacto econômico. Sabe-se que o Brasil permitiu acúmulo de capital, acarretando-se prejuízo das questões relacionadas ao bem-estar da sociedade.

Por fim, é necessário compreender que após finalizar o tratamento, uma paciente com câncer ginecológico, poderá apresentar infertilidade, menopausa prematura, linfedema, disfunções sexuais, intestinais, hormonais ou urinárias, contudo, a 
Organização Mundial da Saúde (OMS), enfatiza que as doenças crônicas não transmissíveis (DCNT), apresentam um grave problema de saúde pública mundial e negligenciadas e as doenças neoplásicas são as que representam um alto percentual de óbitos no Brasil (ALVES et al., 2012).

Deste modo, este estudo teve como metodologia utilizada a de pesquisa bibliográfica, de natureza descritiva e abordagem qualitativa, com coleta de dados realizada em janeiro de 2021, utilizando como base de dados o Google Scholar e as palavraschaves: Estado de bem-estar social; Qualidade de vida; Câncer Ginecológico. " Após busca dos estudos foi utilizado o recorte temporal de 10 anos pela escassez de publicação disponível e selecionados apenas artigos publicados em periódicos científicos, consistindo na fonte mais fidedigna de divulgação do conhecimento científico e com exceção de estudos que possuírem dados de extrema relevância para a pesquisa.

\section{DESENVOLVIMENTO}

\subsection{POLÍTICA PÚBLICA E O WELFARE STATE}

De acordo com Lynn (1980) Política Pública é definida como um conjunto de ações do governo que produz efeitos específicos. Efeitos esses que definem diretamente a tomada de decisão, por parte direcionada dos governantes. Para que a política seja implementada, a tomada de decisão não é técnica, nem mesmo racional, muitas vezes essa definição é determinada por questões políticas, implicando diretamente na sociedade.

Essa organização, baseada na proteção de trabalhadores assalariados e contra possíveis riscos aos trabalhadores em uma economia participativa de mercado é que dão sinais de primeiro entendimento sobre Welfare State[11] (KERSTENETZKY, 2012).

RC: 89583

Disponível em: https://www.nucleodoconhecimento.com.br/saude/cancer-ginecologico 
O estado de bem-estar social, apresenta diversos conceitos, dimensões e modelos iniciado na década de 1940, apresenta em seus fundamentos aspectos relevantes para a sociedade no século XXI, contudo, à diversos grupos que carecem de seus princípios para uma sociedade mais justa e igualitária.

Em continuidade, Arretche (1995) alude que o direito social e a solidariedade equivalem-se aos princípios reguladores da vida social. Os sistemas de proteção social originaram-se a partir da necessidade fundamental de eliminar ou diminuir o impacto de determinados riscos sobre o indivíduo e sociedade, sendo consequência da ação pública que tem por objetivo preservar a sociedade de efeitos de riscos clássicos: desemprego, exclusão por (renda, raça, etnia, cultura), doença e invalidez (VIANA; LEVCOVITZ, 2015).

Dias e Matos (2015), descrevem que as ações sociais que ocorriam na esfera privada estavam ligadas a iniciativas da Igreja ou de organizações da sociedade, motivadas com a ética e a moral até 1940, porém estas ações não ocorriam de forma sistêmica sendo de forma desorganizada e irregular. As legislações e medidas de proteção social surgiram no final do século XIX consolidando-se entre (1939 - 1945), após a Segunda Guerra Mundial.

Segundo Rodrigues (2010) as medidas de Política Social e econômica, convertem-se em direitos sociais assegurados pelo Estado aos indivíduos, destacando: as provisões de serviços em especial a segurança no mercado de trabalho garantida por (condições de trabalho, salário, representação dos interesses trabalhistas), a proteção contra riscos da vida social (saúde, educação e habitação) e as garantias de renda (segurodesemprego, auxílio-família e auxílio-doença).

Todavia, para Rodrigues (2013), o Estado de bem-estar social de um país é proveniente de sua construção histórica, onde a conquista da cidadania vem por meio do desenvolvimento industrial do país e quanto maiores estes desenvolvimentos, tornaram-se também maiores os investimentos com Welfare State.

$\mathrm{RC}: 89583$

Disponível em: https://www.nucleodoconhecimento.com.br/saude/cancer-ginecologico 
De forma geral, é enfática a percepção de que Secchi (2016) destaca que a dificuldade encontrada na teoria de Bozeman e Pandey é de que as políticas públicas podem ser alteradas em essência, ao longo do ciclo de política pública que varia de acordo com as demandas da sociedade.

\subsection{CONCEITO DE WELFARE STATE}

O Welfare State assim conceituado após o fim da Segunda Guerra Mundial, passou a ser um regime específico de proteção social, onde as políticas sociais e regimes de proteção eram conferidos aos cidadãos e os valores sociais eram provenientes da justiça social e igualdade.

Para Arretche (1995) classifica-se como um fenômeno do século XX, a oferta de serviços sociais para a cobertura de diversas formas de riscos da vida individual e coletiva, no qual, passaram a ser direito assegurado pelo Estado às camadas expressivas da população de países capitalistas desenvolvidas como a Alemanha, por exemplo.

Ademais, Di Giovanni Nogueira (2005) descreve as fases que fizeram parte do processo evolutivo do EBES: A Fase Emergencial do Estado de Bem-estar social foi definida como política social com proteção aos assalariados e a Fase de Consolidação está voltada para as questões financeiras de previdência e gastos dos serviços sociais.

Desta forma, Titmuss (1974), fundamenta sua análise em determinantes de ordem econômica, pois além de se basear sobre os riscos no processo de produção na industrialização, salienta que o Welfare State surge com a necessidade de serviços sociais do Estado.

Outro teórico proeminente é Esping-Andersen, por avaliar com profundidade a proteção social a partir da desmercadorização, ou seja, existe aspectos intrínsecos, variáveis chaves que devem ser contempladas para teorização do EBS (estado de

RC: 89583

Disponível em: https://www.nucleodoconhecimento.com.br/saude/cancer-ginecologico 
bem-estar social) Estado, mercado e democracia que conjuntamente criam-se de forma dependentes e independente da proteção social.

Primero Esping-Andersen (1991), apresenta o surgimento do Welfare State, em dois aspectos a partir da lógica da industrialização, para este teórico a indústria moderna, enfraquece a instituições tradicionais de dependência.

Assim, para fundamentar suas teorias, Esping-Andersen apresenta o primeiro aspecto como sendo a estrutura da industrialização, afirmando que políticas sociais são necessárias em substituição a família, a igreja, mecanismo de proteção ultrapassando os riscos inerentes a vida na industrialização. O segundo aspecto, está na força da mobilização, ou seja, as classes sociais trabalhistas ganham força com equilíbrio de poder para distribuição de renda, conforme o autor social.

Desta maneira, a tipologia de Esping-Andersen apresenta a relevância de estado de bem-estar social a partir de modelos com características políticas, aliado a desmercadorização para sistemas de proteção social.

Para Viana (2015) os processos de modernização e industrialização provém de um momento e regime específico de proteção social, no período pós-guerra.

Wilensky apud Arretche (1995, p.7) em sua descrição, refere-se à Walfare State, como sendo um conjunto de programas governamentais, envolvendo ação estatal, no campo de atenção à saúde, previdência, assistência social, executando-se educação e habitação.

Por fim, entende-se que o Estado não seria encarregado apenas de problemas coletivos, mas de cada pessoa em particular. Para dar concretude a essa concepção, construíram-se grandes sistemas públicos de saúde e proteção social (welfare state), financiados com orçamento estatal e ordenados segundo a lógica da eficácia e eficiência, em confronto com interesses corporativos e sindicais que tradicionalmente

RC: 89583

Disponível em: https://www.nucleodoconhecimento.com.br/saude/cancer-ginecologico 
haviam se incorporado ao mercado. Esse esforço produziu uma nova cultura, inaugurando outro modo de gestão, planejamento e atenção à saúde.

\section{ESTADO DE BEM-ESTAR SOCIAL NO BRASIL}

Assim como Bismark na Alemanha, e Vargas no Brasil, acontece o início do estado de bem-estar social, porém existe a necessidade de se fazer uma passagem anterior a Vargas, para melhor compreensão sobre proteção social no Brasil.

Com três décadas de República, o Brasil pouco conhecia sobre proteção social, em sua reforma de política acanhada, "república reacionária", indiferente a incipiente classe operária de ex-escravos no processo industrial, nada se vislumbrava sobre proteção social, tanto em relação as condições humanas de vida, como condições de trabalho (KERSTENETZKY, 2012).

Deste modo, a partir do século XX, entre 1930 e 1970, com afastamento de produção agrícola, e modernização da economia industrial e urbana, através de exportações primárias, aumenta a intervenção do Estado entre capital, trabalho e desenvolvimento, porém relacionado a proteção social, como direitos, podem ser interpretadas como fragmentada e seletiva (DRAIBE, 1993), não se evidencia ainda uma cobertura contra os riscos sociais.

Assim sendo, o início de proteção social brasileiro, se inicia nesta época, em uma dimensão autoritária, através da organização dos trabalhadores incipientes e a organização da economia, diminuindo a legitimidade das lideranças trabalhista, iniciando pela modernização.

Nesse sentido, Kerstenetzky (2012), afirma que; o marco teórico do estado de bemestar social brasileiro, principia com a Lei dos Acidentes de Trabalho (facultativo), em 1919, para indenização de operários em caso de acidentes e a Lei Eloy Chaves de 1923, que estabelece a criação da Caixa de Aposentadorias e Pensões (CAP) para trabalhadores ferroviários, sendo para esta teórica: "modelo sugerido para ferroviários

RC: 89583

Disponível em: https://www.nucleodoconhecimento.com.br/saude/cancer-ginecologico 
se torna um template a ser aplicado nas empresas de diferentes categorias" (KERSTENETZKY, 2012, p. 187).

\section{PERIODIZAÇÃO OU FASES: DO GOV. VARGAS ATÉ LULA (1930-} 2010)

\subsection{SEGUNDA REPÚBLICA ERA VARGAS (1930 A 1945)}

Getúlio Vargas, inicia-se a fase de forte centralização política e participação estatal nas políticas públicas criada em 1942. A Fundação Especial de Saúde Pública (SESP) desenvolveu ações de saúde pública, onde a Política de Saúde adotada reforça ações de caráter coletivo sob a gestão do Ministério da Saúde e Educação, separadas de ações curativas e individuais vinculadas ao IAPS, reforçando a dualidade do modelo assistencial (DIAS; MATOS, 2012).

\subsection{REDEMOCRATIZAÇÃO (1945 A 1963)}

A fase denominada redemocratização, conforme Kerstenetzky (2012), iniciou-se em 1945, marcado pelo final da Segunda Guerra Mundial, com a deposição de Vargas, dando-se em 1946 o início do Governo de Gaspar Dutra, onde no ano de 1948 lançou o Plano Salte (Saúde, Alimentação, Transporte e Energia) aprovado em 1950. Sendo criado em 1953 o Ministérios da Saúde, independente da área da Educação no Governo de Vargas que retornou em 1954, e no então governo de Juscelino Kubitscheck houve a ampliação do modelo médico assistencial privatista, que perdurou dos anos 60 aos 80 .

\subsection{REGIME MILITAR (1964 A 1984)}

Seguindo uma perspectiva histórica Dias e Matos (2012) retrata que o regime militar foi retratado pelo período de crise política econômica, que perdurou de (1864 a 1984) onde no campo de saúde implantou-se de modo gradual um sistema de saúde 
caracterizado pelo predomínio financeiro e burocracia técnica das instituições previdenciárias, onde se priorizava a mercantilização da saúde.

Sendo assim, em 1966 promoveu-se a unificação dos Institutos de Aposentadorias e Pensões (IAPs), com a criação do Instituto Nacional de Previdência e Assistência Social (INPS), subordinado ao Ministério do Trabalho e Previdência Social, sendo responsável pelos benefícios previdenciários e pela assistência Médica aos assegurados e seus familiares.

\subsection{NOVA REPÚBLICA (1985 A 1988)}

Neste período, José Sarney toma posse na Presidência da República e mantém o compromisso assumido por Tancredo Neves, período em que o cenário de redemocratização da sociedade com movimentos sociais estimula a participação popular. Na ocasião, o quadro sanitário evidenciava a redução da mortalidade infantil e doenças imunopreviníveis, aumento da mortalidade por conta da AIDS e o surgimento da Dengue em algumas capitais (DI GIOVANNI NOGUEIRA, 2005).

Ademais, em 1988, é promulgada a Constituição Federal, no qual, foi aprovado o Sistema Único de Saúde (SUS), incorporado à proposta do movimento da Reforma Sanitária apresentada por emenda popular, significando grande vitória da sociedade.

Por fim, um dos principais programas universalistas que vai de encontro ao conceito do estado de bem-estar social (EBS), é o Programa Saúde da Família (PSF), que possibilita através de seus mecanismos a proteção aos riscos inerentes à vida na contemporaneidade, ou seja, a toda a família, embora tenha existido uma desigualdade espacial, este em suma é um programa que ampara pessoas fora do mercado, com necessidade especiais e idosos (KERSTENETZKY, 2012).

RC: 89583

Disponível em: https://www.nucleodoconhecimento.com.br/saude/cancer-ginecologico 


\section{A SAÚDE COMO DIREITO LEGAL E COMO POLÍTICA DO ESTADO}

Pela Constituição Brasileira, em seu artigo 196 a saúde é um direito de todos e dever do Estado, garantido por meio de políticas sociais e econômicas que visem a redução do risco de doença e de outros agravos e ao acesso universal e igualitário as ações e serviços para sua promoção, proteção e recuperação.

Aguiar, (2015) traz em sua obra a definição do Sistema Único de Saúde (SUS), estabelecido formalmente a partir da Constituição Federal de 1988, composto pelo conjunto organizado e articulado de ações de saúde, integrante das organizações públicas de saúde das esferas municipal, estadual e federal.

Dessa maneira, Aguiar, 2015 descreve que a evolução da Política de saúde teve seu início a partir da estreita relação com a evolução da política econômica e social da sociedade brasileira, obedecendo a ótica do capitalismo nacional, influenciado pelo capitalismo internacional.

\subsection{QUALIDADE DE VIDA E A BUSCA DE INDICADORES SOCIAIS}

Alguns problemas conceituais em qualidade de Vida, partir do início do século XX, levaram a uma crescente busca de indicadores de riqueza e de desenvolvimento, como o produto Interno Bruto (PIB), a renda per capita e o Índice de mortalidade Infantil.

Recentemente medidas de alta complexidade, como o Índice de Desenvolvimento Humano (IDH), elaborado pelo programa das Nações Unidas (ONU) para o desenvolvimento, procuraram incluir aspectos de natureza social e cultural como: indicadores de saúde e educação às tradicionais medidas econômicas (FLECK, 2008).

RC: 89583

Disponível em: https://www.nucleodoconhecimento.com.br/saude/cancer-ginecologico 


\subsection{POLÍTICAS PÚBLICAS DE ATENÇÃO ONCOLÓGICA VOLTADAS AO CÂNCER DE COLO DE ÚTERO}

Sabe-se que o câncer é reconhecidamente um problema de saúde pública, todavia, possui uma política de cobertura nacional voltada para a sua especificidade, a Política Nacional para prevenção e Controle do Câncer, que possui diretrizes relacionadas à promoção da saúde e à prevenção nos diferentes níveis, incluindo o controle com ações de promoção a saúde.

Sob a perspectiva histórica, as políticas de saúde a mulher tiveram sua descoberta no início do século XX, somente focalizando a assistência à mulher no processo de reprodução, principalmente mediante ações voltadas ao pré-natal, parto e puerpério.

Sendo assim, em 1984, de fato foi instituído o Programa de Atenção Integral à Saúde da Mulher (PAISM), com a finalidade dos serviços básicos de saúde oferecer às mulheres atividades de prevenção do câncer do colo do útero. A principal contribuição desse Programa foi introduzir e estimular a coleta de material para o exame citopatológico como procedimento de rotina na consulta ginecológica, tornando-se a primeira ação de âmbito nacional do Ministério da Saúde para prevenção do câncer do colo do útero (BRUAN et al., 2016).

Ademais, em 21 de junho de 1998, o Ministério da Saúde fundou o Programa Nacional de Combate ao Câncer de Colo do Útero através da Portaria GM/MS № 3040/98, com medidas para estruturação da rede assistencial, estabelecimento de um sistema de informações para o monitoramento das ações (SISCOLO) e dos mecanismos para mobilização e captação de mulheres, assim como definição das competências nos três níveis de governo, momento em que mais de três milhões de mulheres foram mobilizadas para fazer o exame citopatológico.

Entretanto, é competência de os órgãos federais, estaduais e municipais organizarem a Rede Pública de saúde no cumprimento da Portaria para controle do câncer do colo do útero nos aspectos envolvendo a promoção, prevenção, detecção precoce, RC: 89583

Disponível em: https://www.nucleodoconhecimento.com.br/saude/cancer-ginecologico 
diagnóstico, tratamento e pós tratamento de forma apropriada (BRAUN; CASTILHOS e DE LIMA, 2016).

Nota-se também que todos os estabelecimentos de saúde que prestam atendimento às pessoas com câncer, possuam infraestrutura adequada, recursos humanos capacitados, recursos materiais, equipamentos e insumos suficientes, de maneira a garantir o cuidado necessário. Além disso, os gestores devem garantir atuação territorial; a formação e a qualificação dos profissionais e dos trabalhadores de saúde; monitorar e avaliar a cobertura, produção, desempenho e qualidade dos serviços de prevenção e de controle do câncer no país no âmbito do SUS, entre outras.

Assim sendo, para efetividade das políticas, programas e as ações planejadas para prevenir o câncer entre populações, é preciso garantir que todos os profissionais envolvidos no processo sejam parceiros no planejamento e na execução das políticas. É necessário que os formuladores de políticas estabeleçam metas e especifiquem as expectativas que sejam de longo prazo e realísticas (BRAUN; CASTILHOS e DE LIMA 2016).

Contudo, compreende-se que o gestor de saúde em todos os níveis têm a responsabilidade de exercer as funções de coordenação, articulação, negociação, planejamento, acompanhamento, controle, avaliação e auditoria dos recursos humanos, financeiros e materiais empregados na produção de serviços de saúde objetivando sanar as falhas e definir novos planos em prol da população.

\section{CONSIDERAÇÕES FINAIS}

Diante do estudo apresentado, conclui-se que este trabalho alcançou o objetivo proposto de conceituar as teorias, os períodos e fases do Welfare State, e o desenvolvimento do Estado de Bem-Estar Social e tratando-se das políticas públicas em oncologia, nota-se que tais políticas possibilita a reabilitação e reinserção das mulheres na vida social promovendo qualidade de vida.

$\mathrm{RC}: 89583$

Disponível em: https://www.nucleodoconhecimento.com.br/saude/cancer-ginecologico 
O Estado de bem-estar social estava voltado às necessidades sociais e proteção dos cidadãos com a implementação das políticas e programas, no qual, seu início ocorreu na era Vargas na década de 30 , período em que as primeiras políticas sociais eram voltadas para a classe trabalhadora.

Complementarmente, identificou-se que a inclusão de trabalhadores assalariados no sistema de proteção social brasileiro está ligada a expansão da indústria, até mesmo devido um aumento da demanda dos trabalhadores.

Entende-se que as políticas relacionadas ao Câncer de colo de útero por meio de ações governamentais proporcionam estratégias essenciais para a garantia dos direitos e cidadanias das mulheres com câncer de colo de útero em todos os níveis de tratamento além também de pessoas com câncer.

Contudo, com base no que foi observado em meio a condução deste estudo, conceituou-se as políticas do SUS e sua trajetória em prol da assistência à saúde dos cidadãos e abordou-se as questões de previdência e seguridade social como direitos adquiridos e assegurados por lei e que diante das perspectivas da política nacional Oncológica, o INCA (Instituto Nacional de Câncer) estabelece por meio da atenção terciária através dos serviços credenciados ao Sistema Único de Saúde, o acesso universal a assistência integral as mulheres acometidas pelo câncer ginecológico.

\section{REFERÊNCIAS}

ALVES, Pricilla Cândido et al. Conhecimento e expectativas de mulheres no préoperatório da mastectomia. Rev Escola de Enfermagem Usp, Online, v. 44, n. 4, p.989995, mar. 201

ARRETCHE, M.T.S. Emergência e Desenvolvimento do Welfare State: teorias explicativas. Boletim Informativo e Bibliográfico em Ciências Sociais. v. 39, p.340, 1995

RC: 89583

Disponível em: https://www.nucleodoconhecimento.com.br/saude/cancer-ginecologico 
BRAUN, Josiane Bizzi Schlemmer; CASTILHOS, Lívia Gelain; DE LIMA, Suzinara Beatriz Soares. POLÍTICAS PÚBLICAS E A ATUAÇÃO DOS GESTORES FRENTE AO CÂNCER DE MAMA E DO COLO UTERINO. Saúde (Santa Maria), p. 53-62, 2016.

DI GIOVANNI, Geraldo; NOGUEIRA, marco Aurelio (Org). Dicionário de Políticas Públicas. $2^{a}$ ed. São Paulo: Editora Unesp/Fundap, 2015.v. 1065p.

DIAS, Reinaldo; MATOS, Fernanda. Políticas Públicas: Princípios, propósitos e processos. São Paulo: Atlas, 2012.

DRAIBE, Sônia. Welfare State no Brasil: Características e Perspectivas. Caderno de Pesquisa, n.8, pp.1-50, Campinas, NEPP-Unicamp, 1993.

ESPING-ANDERSEN, Gosta. As três economias políticas do Welfare State. Lua Nova, n. 24, pp.85-116, set./1991.

FLECK, 2008. A avaliação de qualidade de vida: guia para profissionais da saúde/ Marcelo Pio de Almeida Fleck... [et al.]. -Porto Alegre: Artmed, 2008

INSTITUTO NACIONAL DE CÂNCER. ABC do câncer: abordagens básicas para o controle do câncer. 2011.

KERSTENETZKY, Celia Lessa. O Estado do Bem-Estar Social na Idade da Razão. Rio de Janeiro: Elsevier, 2012.

MARSHALL, Thomas H. Cidadania, classe social e status. Tradução de Merton Porto Gadelha. Rio de Janeiro: Zahar, 1967.

RODRIGUES, M.A. Políticas Públicas. (Coleção Folha Explica). São Paulo: Publifolha Editora, 2010.

SECCHI, Leonardo. Políticas Públicas: conceitos, esquemas de análise, casos práticos. São Paulo: Cengage Learning, 2013. RC: 89583 
https://integrada.minhabiblioteca.com.br/\#/books/9788522114085/cfi/0!/4/2@100:0.0 0

VIANA, A.L.D., LEVCOVITZ, E. Proteção social: introduzindo o debate. In: VIANA, A.L.D., ELIAS, P.E.M., IBAÑEZ, N. Proteção social: dilemas e desafios. São Paulo: Hucitec, 2005. p.15-57.

\section{APÊNDICE - REFERÊNCIA DE NOTA DE RODAPÉ}

11. Oxford English Dictionary, Welfare State significa: "um país no qual o bem-estarsocial dos membros da comunidade é garantido por meio de serviços sociais organizados pelo Estado" (KERSTENETZKY, 2012, p. 2).

Enviado: Abril, 2021.

Aprovado: Junho, 2021.

$\mathrm{RC}: 89583$

Disponível em: https://www.nucleodoconhecimento.com.br/saude/cancer-ginecologico 